\title{
Protective effect of vascular endothelial growth factor/vascular permeability factor 165 and 121 on glomerular endothelial cell injury in the rat
}

\author{
Kenji Miyamoto ${ }^{1}$, Yasunori Kitamoto ${ }^{1}$, Hiroshi Tokunaga ${ }^{2}$, Motohiro Takeya ${ }^{3}$, Taichi Ezaki ${ }^{4}$, \\ Takahisa Imamura ${ }^{5}$ and Kimio Tomita $^{1}$ \\ ${ }^{1}$ Department of Nephrology; ${ }^{2}$ Department of Metabolic Medicine; ${ }^{3}$ Department of Cell Pathology, Graduate \\ School of Medical Sciences, Kumamoto University, Kumamoto, Japan; ${ }^{4}$ Department of Anatomy and \\ Developmental Biology, Tokyo Women's Medical University, Tokyo, Japan and ${ }^{5}$ Department of Molecular \\ Pathology, Graduate School of Medical Sciences, Kumamoto University, Kumamoto, Japan
}

\begin{abstract}
Vascular endothelial growth factor/vascular permeability factor (VEGF/VPF) promotes the repair of injured vessels by stimulating angiogenesis. VEGF/VPF reportedly has cytoprotective activity but no study has shown the protective effect of VEGF/VPF on glomerular endothelial cells. We examined whether recombinant VEGF/ VPF121 and VEGF/VPF165 isoforms could prevent injury of glomerular endothelial cells. Mild glomerular injury was induced in rats by an intravenous-injection of a limited dose of anti-Thy-1.1 antibody to obtain lesions similar to those found in the human disease. Recombinant VEGF/VPF165, VEGF/VPF121 or BSA was administered $4 \mathrm{~h}$ before the injection of the antibody, and once daily for 3 days. In the BSA-injected rats, mesangial cell lysis and endothelial cell injury in dilated capillary tufts were evident without endothelial cell apoptosis on days 1-4. Thereafter, cell proliferation and repair began and remodeling of the glomeruli was completed by day 28 . Macrophages but not polymorphonuclear leukocytes accumulated significantly in the glomeruli on days 1-4. Treatment with VEGF/VPF isoform protected endothelial cells but not mesangial cells from destruction on day 1, and accelerated the repair of both types of cells, which was completed by day 18, 10 days earlier than that of the control animals. The results indicate that VEGF/VPF121 or VEGF/VPF165 can protect glomerular endothelial cells against injury, independent of apoptosis-inhibition activity, thereby promoting reconstruction of glomeruli. The protective effect of VEGF/VPF on endothelial cells suggests that it could provide therapeutic benefit for certain kidney diseases.

Laboratory Investigation (2004) 84, 1126-1136, advance online publication, 14 June 2004; doi:10.1038/labinvest.3700134
\end{abstract}

Keywords: VEGF/VPF165; VEGF/VPF121; endothelial protection; repair; glomerulonephritis

Vascular endothelial growth factor/vascular permeability factor (VEGF/VPF) mediates angiogenesis and vasculogenesis, and regulates vascular permeability. ${ }^{1}$ There are six VEGF isoforms in human beings, VEGF/VPF121, 145, 165, 183, 189 and 206, ${ }^{1}$ and at least three isoforms in mice, VEGF/VPF120, 164 and $188 .^{2}$ The isoforms have different affinities for VEGF/VPF receptors, ${ }^{3,4}$ heparan sulfate $^{5}$ and coreceptor, neuropilin- $1,{ }^{6}$ and they are expressed at different levels in different tissues in both

Correspondence: Associate Professor Y Kitamoto, MD, PhD, Department of Nephrology, Graduate School of Medical Sciences, Kumamoto University, Honjo 1-1-1, Kumamoto 860-8556, Japan. E-mail: yasunori@kaiju.medic.kumamoto-u.ac.jp

Received 21 September 2003; revised 26 April 2004; accepted 1 May 2004; published online 14 June 2004 embryos and adults. ${ }^{2}$ In human glioma cells, there was a difference in angiogenesis induced by overexpression of each isoform. ${ }^{7}$ Glioma cells show increased vascular density in intracranial space by overexpressing either VEGF/VPF165 or VEGF/ VPF189 but not VEGF/VPF121. Mice expressing only VEGF/VPF120 showed impaired cardiac $^{8}$ and renal angiogenesis. ${ }^{9}$ Thus, it is likely that VEGF/VPF isoforms have distinct activities.

In the kidney, glomerular endothelial cells are continuously exposed to high blood pressure for blood filtration, and are continuously destroyed and regenerated physiologically. ${ }^{10}$ When the endothelial cells are severely injured, defective repair leads to glomerular sclerosis. ${ }^{11}$ Under such conditions, endothelial cells rely on various protective mechanisms to survive. One well-known strategy involves 
VEGF/VPF signaling. ${ }^{12-16}$ Although the angiogenic effect of VEGF/VPF165 has been studied during the repair of glomerular injury, ${ }^{17,18}$ glomerulonephritis models used in these studies show excessive glomerular destruction, which differs greatly from the changes observed in human disease. It was reported recently that VEGF/VPF plays a so-called nonangiogenic role ${ }^{19}$ to protect endothelial cells, ${ }^{20,21}$ neural cells $^{22}$ and the liver $^{23}$ against injury. It is important to study the VEGF/VPF effect on glomerular endothelial cells and the mechanism in a biologically relevant model.

Therefore, we induced relatively mild glomerular injury by injecting a limited dose of Thy-1.1 antibody into rats to obtain endothelial cell changes similar to those seen in human mesangial proliferative glomerulonephritis. We then examined the protective effect of recombinant VEGF/VPF165 and VEGF/VPF121 on the glomerular changes.

\section{Materials and methods}

\section{Animals}

Approval for animal studies was obtained from the Ethics Committee for animal experiments in Kumamoto University. Male 6-week-old Wistar rats, weighing about $160 \mathrm{~g}$, were used for these studies.

\section{Induction of Mesangial Proliferative Glomerulonephritis}

Glomerulonephritis was induced in rats by injecting a mouse monoclonal antibody to Thy-1.1, as a purified IgG (MCA47G, Serotec Ltd, Oxford, England), into jugular veins through indwelling catheters (day 0). The amounts of the antibody varied from 0.06 to $0.25 \mathrm{mg}$ per animal and the induction of glomerular changes initiated by mesangiolysis was assessed. The severity of glomerular changes depended on the dose of injected anti-Thy-1.1 antibody, which was between 0.06 and $0.25 \mathrm{mg}$. Glomerulonephritis induced by injecting $0.20 \mathrm{mg}$ of the antibody caused severe mesangiolysis and capillary rupture (ballooning) in the glomerulus, which may be too severe to simulate histological changes in human mesangial proliferative glomerulonephritis. On the other hand, $0.12 \mathrm{mg}$ of the antibody induced milder but significant glomerular changes, and we selected this dose to induce glomerulonephritis in this study.

\section{Treatment Protocol}

Since the administered anti-Thy-1.1 antibody remains detectable in glomeruli for 3 days, ${ }^{24}$ recombinant VEGF/VPF165 was administered for three consecutive days after the initial anti-Thy-1.1 injection to counteract the nephritogenic activity of the antibody. An amount of $10 \mu \mathrm{g}$ of recombinant VEGF/
VPF165 (Pepro Tech EC Ltd, London, England) or $8 \mu \mathrm{g}$ of recombinant VEGF/VPF121 (TOAGOSEI CO Ltd, Tsukuba, Japan) was dissolved in $0.1 \mathrm{ml}$ of saline and administered into the jugular vein of the animal through the catheter $4 \mathrm{~h}$ before injecting the anti-Thy-1.1 antibody. For experiments with VEGF/ VPF165, $10 \mu \mathrm{g}$ of the protein per dose was additionally administered 1 (day 1), 2 (day 2 ) and 3 days (day 3) after injecting the antibody. As a control, the same amount of BSA on a molar concentration (Sigma Chemical Co., St Louis, MO, USA) was administered instead of VEGF/VPF. Kidneys were harvested under anesthesia on days 1, 4, 18 and 28, and submitted for histological examination. The same animal was used to harvest one kidney on day 4 and the other on day 18. For experiments with VEGF/ VPF121, there were no additional injections and the kidneys were harvested on day 1. Experiments were repeated three times independently for VEGF/ VPF165 and VEGF/VPF121.

\section{Histological Assessment}

\section{Routine histological observation and immunohistochemistry}

Renal tissues were embedded in the OCT compound and rapidly frozen in liquid nitrogen, or were fixed in $4 \%$ paraformaldehyde in phosphate buffer and embedded in paraffin. Paraffin sections of $4 \mu \mathrm{m}$ thickness were stained with either hematoxylin and eosin (HE), periodic acid-Schiff (PAS) or Azan-Mallory. Immunohistochemistry for vWF and proliferating cell nuclear antigen (PCNA) was performed on frozen sections, and staining with $\mathrm{RM}-4,{ }^{25}$ that specifically recognizes rat macrophage/ dendritic cells, was performed on paraffin sections. Frozen sections of $8 \mu \mathrm{m}$ thickness were fixed with $4 \%$ paraformaldehyde and $0.05 \%$ glutaraldehyde in $0.1 \mathrm{M}$ phosphate buffer at room temperature for $10 \mathrm{~min}$, followed by incubation with $0.001 \%$ pepsin in $0.01 \mathrm{~N} \mathrm{HCl}$ at $37^{\circ} \mathrm{C}$ for 5 min for antigen unmasking or retrieval. Paraffin sections of $6 \mu \mathrm{m}$ thickness were preincubated with $0.1 \%$ trypsin (Sigma) in $50 \mathrm{mM}$ Tris-HCl buffer ( $\mathrm{pH} 7.6$ ) at $37^{\circ} \mathrm{C}$ for $15 \mathrm{~min}$ to unmask antigen. Intrinsic peroxidase activity was blocked by treating tissues with $1 \% \mathrm{H}_{2} \mathrm{O}_{2}$ /methanol at room temperature for $20 \mathrm{~min}$. Sections were incubated overnight at $4{ }^{\circ} \mathrm{C}$ with mouse monoclonal anti-PCNA antibody, rabbit anti-human vWF polyclonal antibody, or mouse monoclonal antibody RM-4, followed by DAKO Envision ${ }^{\mathrm{TM}}$ PO or AP system. Bound antibody was visualized by peroxidase reaction in a $3,3^{\prime}$-diaminobenzoic tetrahydrochloride (DAB) (brown) and $\mathrm{H}_{2} \mathrm{O}_{2}$ solution, or by alkaline phosphatase reaction for Fuchsin (DAKO Corp., Carpinteria, CA, USA) (red).

For morphometrical studies, more than 30 crosssections of glomeruli were examined sequentially in each kidney sample. 


\section{Confocal Microscopy}

For detailed observation of PCNA-positive endothelial cells in glomeruli, confocal microscopic analysis was also performed. Frozen sections of $8 \mu \mathrm{m}$ thickness were fixed with $4 \%$ paraformaldehyde and $0.05 \%$ glutaraldehyde in $0.1 \mathrm{M}$ phosphate buffer at room temperature for $10 \mathrm{~min}$, followed by an incubation with $0.001 \%$ pepsin in $0.01 \mathrm{~N} \mathrm{HCl}$ at $37^{\circ} \mathrm{C}$ for $5 \mathrm{~min}$, for unmasking or antigen retrieval. Sections were incubated overnight at $4^{\circ} \mathrm{C}$ with antiPCNA mouse monoclonal antibody and anti-human vWF rabbit polyclonal antibody at a final dilution of 1:100 and 1:10000, respectively. Next, tissue sections were incubated with goat anti-rabbit-Alexa488 antibody (Molecular Probes, Eugene, OR, USA) and goat anti-mouse-Alexa546 antibody (Molecular Probes) at a final dilution of 1:100. The images were collected on a microscope (model IX70; Olympus) attached to a confocal laser scanning device (model Fluoview FV500; Olympus, Tokyo, Japan). The laser lines were $488 \mathrm{~nm}$ (Alexa488) and $543 \mathrm{~nm}$ (Alexa546).

\section{Counts of nuclei and tufts}

The number of nuclei was regarded as the number of cells. The degree of mesangiolysis was determined by the decrease in glomerular cell numbers. The number of nuclei on day 1 was counted in 25 glomeruli at their equatorial sections on HE-stained slides from six kidney samples. Each value was divided by its corresponding glomerular area to compute a relative number of nuclei per glomerulus. The number of tufts on day 1 was also counted in 25 glomeruli from six kidney samples at their equatorial sections on Azan-Mallory-stained slides and a relative number of tufts per glomerulus was similarly determined. Countings were performed by three observers independently and the average values were used as data.

\section{VEGF/VPF Measurement}

Serum samples were stored at $-80^{\circ} \mathrm{C}$ until use and submitted to colorimetric enzyme immunoassay to quantitate VEGF/VPF.

\section{Mesangial proliferation}

On PAS-stained sections, 24 glomeruli from three kidney samples were observed at their equatorial sections at each time point by three observers. The degree of mesangial proliferation was scored as follows: expanded mesangial areas in $\leq 25,26-50$, 51-75 and $76-100 \%$ of the total glomerular area were scored as $0,1,2$ and 3, respectively.

\section{Endothelial cells}

Tissues were made to reacted with anti-human vWF polyclonal antibody (DAKO Corp.), at a final dilution of 1:20000, followed by DAKO Envision ${ }^{\mathrm{TM}}$
PO system, to identify endothelial cells immunohistochemically. The percentage of vWF-positive brown-colored area in glomerulus at its equatorial section was calculated by computer-assisted image analysis using the Mac Scope software (Mitani Corp., Fukui, Japan) in 15 glomeruli from each of three kidney samples per time point.

\section{Proliferating cells}

Proliferating cells in the tissue were stained immunohistochemically using an antibody to proliferating cell nuclear antigen (PCNA) (DAKO Corp.) at a final dilution of 1:200, followed by the DAKO Envision $^{\mathrm{TM}}$ AP system. The number of PCNApositive cells was counted in 30 glomeruli at their equatorial sections in three kidney samples per time point by three observers independently. Each value was expressed as a relative number of proliferating cells per glomerulus. Double staining for vWF and PCNA was also performed.

\section{Polymorphonuclear leukocytes (PMN) and macrophages}

PMN were identified morphologically and counted in 15 HE-stained glomeruli at their equatorial sections in three kidney tissues from three independent experiments at each time point by three observers independently. Macrophage/dendritic cells were stained immunohistochemically using a monoclonal antibody RM-4, at a final concentration of $10 \mu \mathrm{g} / \mathrm{ml}$, followed by the DAKO Envision ${ }^{\mathrm{TM}}$ PO system. RM-4-positive cells were counted in 18 glomeruli at their equatorial sections in the three kidney samples from three independent experiments per time point by three observers independently. Each value was expressed as a relative number of the cells per glomerulus.

\section{Apoptotic endothelial cells}

Apoptotic endothelial cells were detected by TUNEL method using an apoptosis detection kit (ApopTag; Serologicals Corp., Norcross, GA, USA) followed by immunostaining for RECA-1 (SANBIO BV, Uden, Netherlands). Briefly, $4 \mu \mathrm{m}$ paraffinembedded sections were deparaffinized, rehydrated, and treated with proteinase $\mathrm{K}(20 \mathrm{mg} / \mathrm{ml})$ for $15 \mathrm{~min}$. Intrinsic peroxidase activity was blocked with $3 \%$ $\mathrm{H}_{2} \mathrm{O}_{2}$ in PBS for 5 min at room temperature. Sections were incubated in a terminal deoxynucleotidyl transferase reaction mixture containing digoxigenin-dNTP for $1 \mathrm{~h}$ at $37^{\circ} \mathrm{C}$, followed by treatment with antidigoxigenin peroxidase conjugate for $30 \mathrm{~min}$ at room temperature. Apoptosis was visualized with peroxidase reaction for $\mathrm{DAB}$ with $\mathrm{NiCl}_{2}, \mathrm{CoCl}_{2}$ and $\mathrm{H}_{2} \mathrm{O}_{2}$ solution (black). The sections were then incubated with anti-RECA-1 at a final dilution of 1:50, followed by detection using the Envision ${ }^{\mathrm{TM}} \mathrm{AP}$ system (DAKO Corp.). Immunoactivity was visualized by alkaline phosphatase reaction for Fuchsin (red) (DAKO Corp.). 


\section{Electron Microscopy Studies}

For electron microscopic observations, on day 1 small blocks of fresh kidney were fixed with $2.5 \%$ glutaraldehyde for $1 \mathrm{~h}$, washed with $0.1 \mathrm{~mol} / \mathrm{l}$ cacodylate buffer ( $\mathrm{pH}$ 7.4), and fixed further with $1 \%$ osmium tetroxide for $1 \mathrm{~h}$. Then the samples were dehydrated through a graded series of ethanol, and embedded in epoxy resin. Ultrathin sections were examined by an electron microscope (Hitachi H-7500, Tokyo, Japan), with counter staining by uranyl acetate and lead citrate.

\section{Assessment of renal function}

Creatinine concentrations in serum or urine samples were measured using an autoanalyzer, and urinary protein was measured by a pyrogallol red method. The degree of proteinuria was evaluated by the ratio of urinary protein to urinary creatinine.

\section{Western Blotting}

Renal cortical tissues were homogenized by sonication in lysis buffer containing $0.5 \%$ Triton X-100, $0.15 \mathrm{M} \mathrm{NaCl}, 10 \mathrm{mM}$ HEPES, $0.1 \mathrm{mg} / \mathrm{ml}$ aprotinin, $0.1 \mathrm{mg} / \mathrm{ml}$ leupeptin and $1 \mathrm{mM}$ phenylmethanesulfonyl fluoride. Homogenates were mixed by rotation for $2 \mathrm{~h}$ at $4^{\circ} \mathrm{C}$ to extract membrane proteins and were centrifuged at 15000 r.p.m. for $30 \mathrm{~min}$ at $4{ }^{\circ} \mathrm{C}$ to get supernatants. The supernatants containing the same amount of proteins were electrophoresed on a $7.5 \%$ sodium dodecyl sulfate polyacrylamide gel, and blotted to a membrane. Flk-1 was immunologically detected with a specific polyclonal antibody (SC504, Santa Cruz Biotechnology, Santa Cruz, CA, USA).

\section{Statistics}

All data were reported as mean \pm standard deviation. Data were analyzed by ANOVA.

\section{Results}

\section{Effects of VEGF/VPF Treatment on the Course of Glomerulonephritis}

After injection of anti-Thy-1.1 antibody, mesangiolysis was observed and capillary tuft fusions appeared on day 1 (Figure 1b) and these glomerular injuries were more evident on day 4 (Figure 1c). Thereafter, the repair process began and mesangial cell proliferation with an increased matrix was clearly evident on day 18 (Figure 1d). By day 28, remodeling of injured glomeruli was completed and glomerular cellularity, mesangial expansion and tuft shapes were similar to those observed before antiThy-1.1 antibody injection (Figure 1e). Ballooning caused by severe capillary injury in glomeruli was scarcely seen throughout the course of the disease. On observation by electron microscopy, on day 1, glomerular capillary tufts were expanded, ruptured and fused to each other (Figure 2a): no apoptotic endothelial cells were observed (Figure 2c). Administration of VEGF/VPF165 reduced the glomerular capillary damage but mesangiolysis was still present
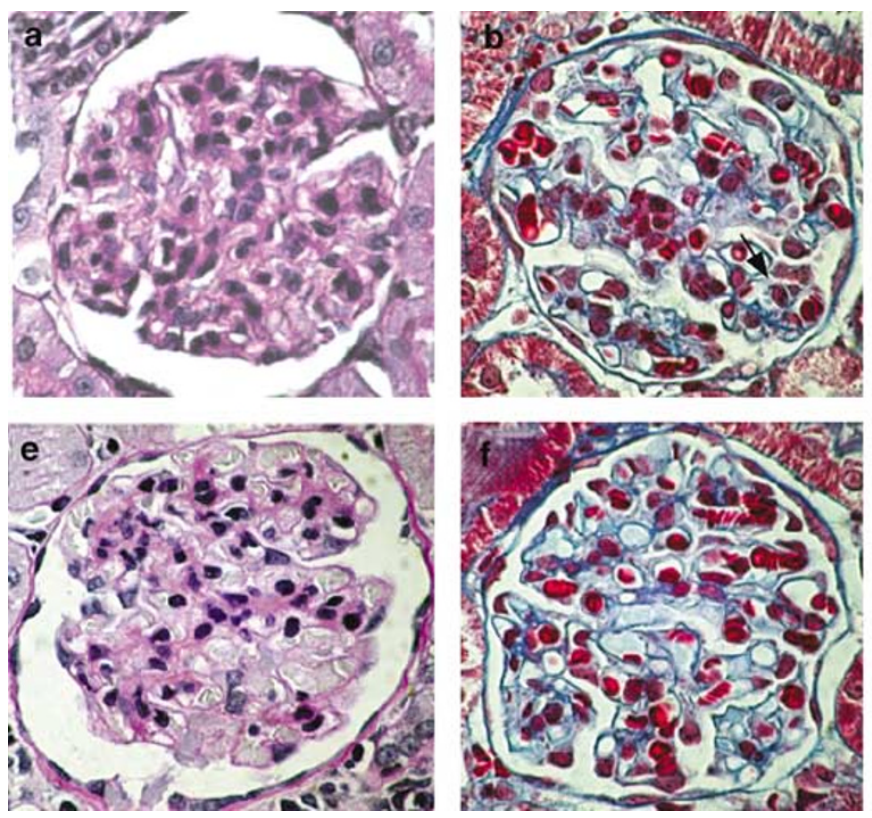
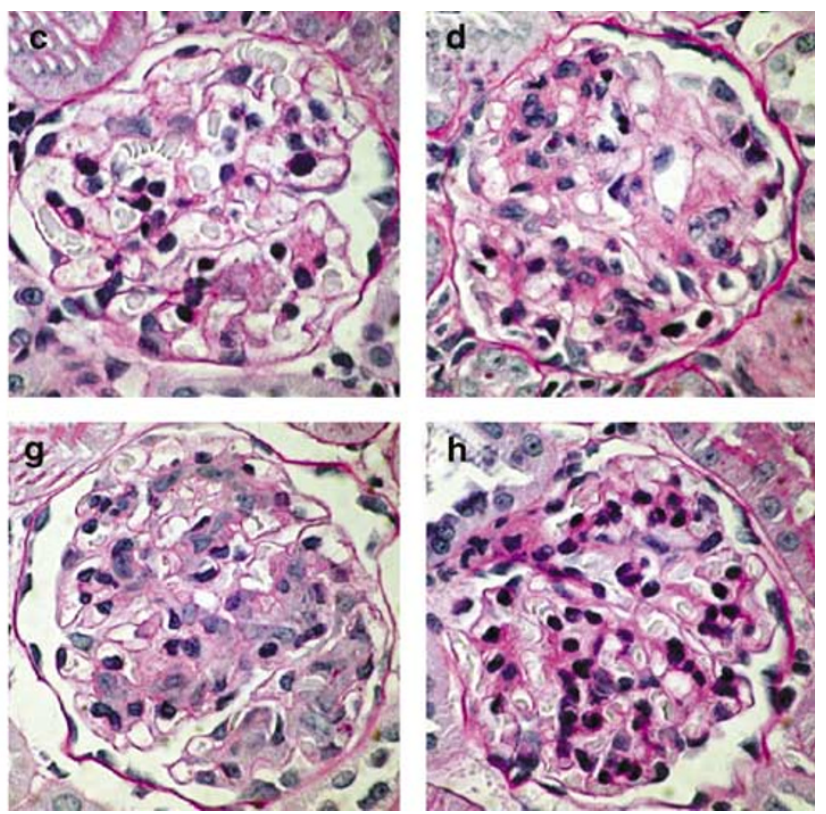

Figure 1 Histological changes along the course of glomerulonephritis in the control (a-e) and the VEGF/VPF-treated (f-h) animals Representative glomeruli are shown before antibody injection (a), on day 1 (b, f), on day $4(\mathbf{c}, \mathbf{g})$, on day 18 (d, h) and on day 28 (e). A representative tuft fusion is indicated by the arrow in (b). Many tuft fusions are evident in (c). (a, c, d, e, g, h) PAS staining. (b, f) AzanMallory staining (magnification $\times 200$ ). 

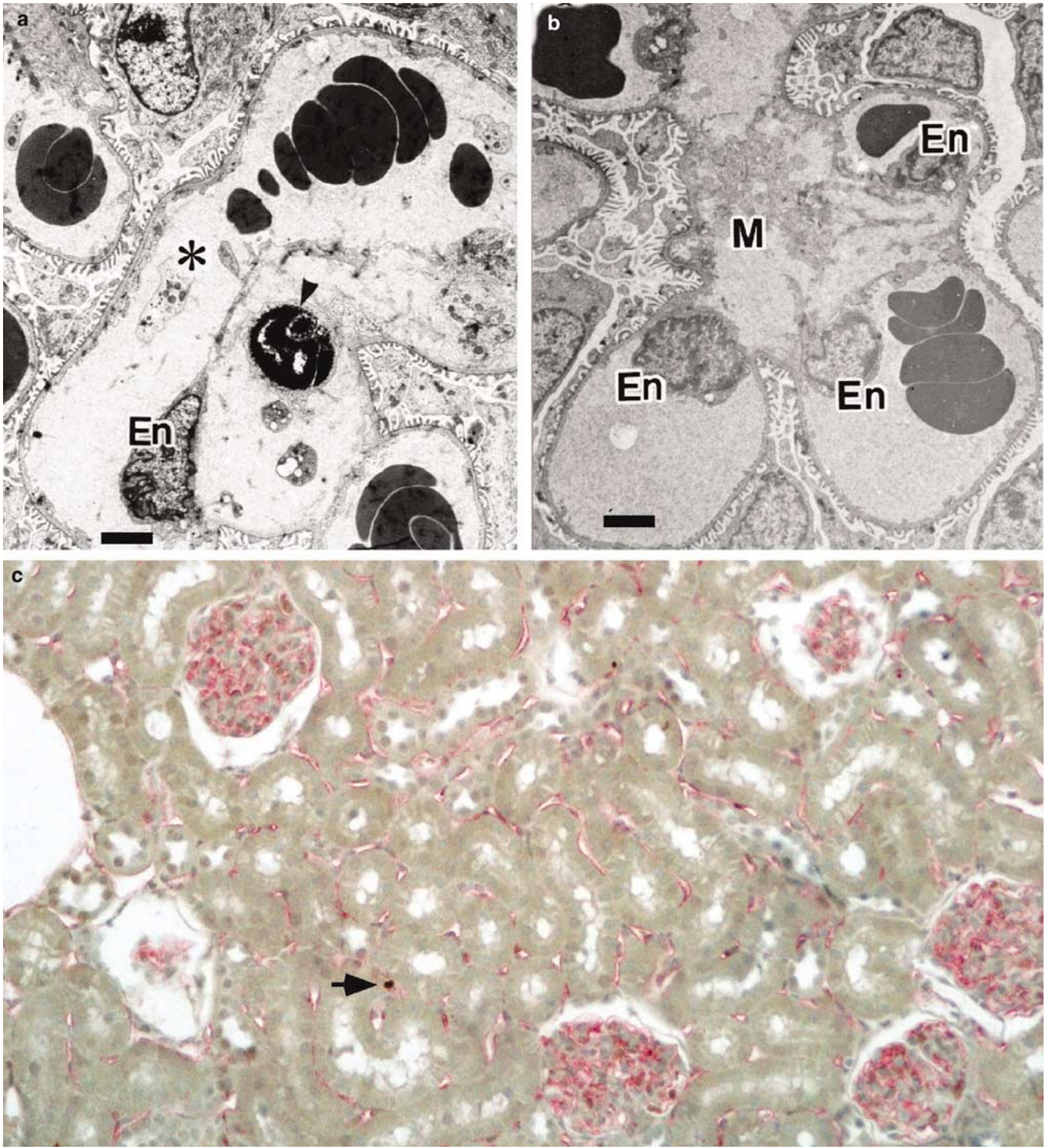

Figure 2 Ultrastructure (a, b) and TUNEL staining (c) of glomeruli on day 1 of glomerulonephritis. (a) A representative glomerulus of the control animal. Mesangiolysis and fusion of tufts $\left({ }^{*}\right)$ by rupture of endothelial cells (En) are shown. The arrowhead shows an apoptotic cell. (b) A representative glomerulus of the VEGF/VPF-treated animals. Although mesangiolysis (M) is evident, endothelial cells (En) are not ruptured. Bars represent $2 \mu \mathrm{m}$. (c) Double staining of the tissue of control glomerulonephritis. Endothelial cells are stained by antiRECA-1 antibody (red) and an apoptotic cell, shown by the arrow, is stained by TUNEL (black). No apoptotic endothelial cells are found in the glomeruli (magnification $\times 100$ ).

on day 1 (Figure 1f). On day 4, mesangial cell proliferation was already evident (Figure 1g). Thereafter, mesangial proliferation was attenuated and on day 18 the glomerular structure was similar to that before anti-Thy-1.1 antibody injection (Figure 1h). On observation by electron microscopy, on day 1, capillary tufts were protected from rupture by VEGF/VPF121 treatment (Figure 2b). Similar 

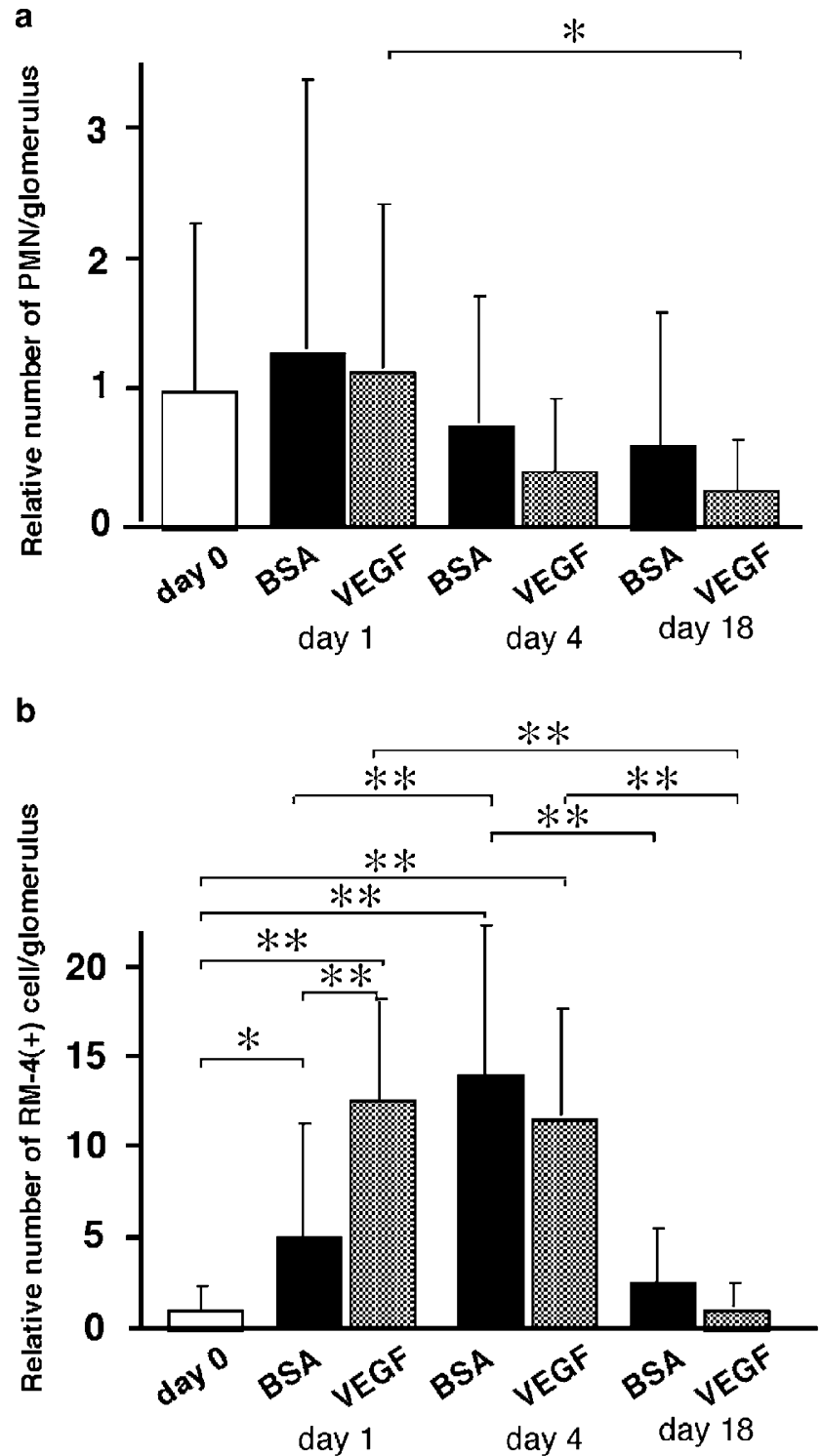

Figure 3 PMN (a) and macrophage (b) accumulations in the glomeruli of animals with BSA or VEGF/VPF165 treatment. ${ }^{*} P<0.05,{ }^{*} P<0.01$.

ultrastructural changes were observed with VEGF/ VPF165 treatment (data not shown). Accumulation of PMN in the glomeruli was negligible through the course of glomerulonephritis (Figure 3a). In control and VEGF/VPF165-treated glomerulonephritis, RM$4(+)$ macrophage accumulation in the glomeruli was evident on days 1 and 4 (Figure $3 \mathrm{~b}$ ). On day 1 , more macrophages had accumulated in the glomeruli of the VEGF/VPF-treated animals than in the untreated controls.

\section{Effect of VEGF/VPF Treatment on Glomerular Changes on Day 1}

\section{Mesangiolysis}

On day 1, the number of cells per glomerulus was decreased and neither VEGF/VPF165 nor VEGF/
VPF121 treatment was significantly protective (Figure $4 a, b)$. Subtracting the sum of PMN and macrophage counts from the total nuclear counts did not alter this result.

Capillary injury

Since capillary tufts are supported by the mesangium, mesangiolysis results in dilation or fusion of capillary tufts and a decrease of the tuft number in the glomerulus. In the control group, the tuft number decreased (Figure 4c). In the VEGF/ VPF165-treated animals, the decrease in tuft number was less than that observed in the control group. Essentially, the same result was obtained when VEGF/VPF121 was used instead of VEGF/VPF165 (Figure 4d).

\section{Effect of VEGF/VPF on Glomerular Repair and Cellular Proliferation}

\section{Mesangial proliferation}

The score of mesangial proliferation was defined as 1.0 before the anti-Thy-1.1 antibody injection (Figure 5a). In the control group, the mesangial proliferation score remained at a low level on day 4, but it increased 2.6-fold on day 18 (Figure 5a). With VEGF/VPF165 treatment, the score increased already 2.1-fold higher than that of the controls on day 4, and decreased to the initial level by day 18 (Figure 5a).

\section{Endothelial cell proliferation}

In the control animals (Figures $5 \mathrm{~b}$ and $6 \mathrm{~d}, \mathrm{j}$ ), the area composed of endothelial cells had increased by 1.6fold on day 4 and remained at the same level through day 18. With VEGF/VPF165 treatment (Figures $5 \mathrm{~b}$ and $6 \mathrm{~g}, \mathrm{~m}$ ), the endothelial area increased by about 2.3 -fold on day 4 but returned to almost the initial level on day 18. The endothelial area of VEGF/VPF-treated animals was larger than that of the control animals on day 4 , but smaller on day 18.

\section{Proliferating cells}

In the control group (Figures 5c and 6e, k), PCNApositive cells increased by about two-fold on day 4 , and returned to almost the initial level on day 18 . With VEGF/VPF165 treatment (Figures 5c and $6 \mathrm{~h}, \mathrm{n})$, the number of PCNA-positive cells increased about three-fold on day 4, but it had decreased to a level much lower than the initial level on day 18.

\section{VEGF/VPF Concentration in the Blood}

Serum VEGF/VPF concentration was under detection limit $(<30 \mathrm{pg} / \mathrm{ml})$ throughout the course of glomerulonephritis both in control and VEGFtreated animals. 

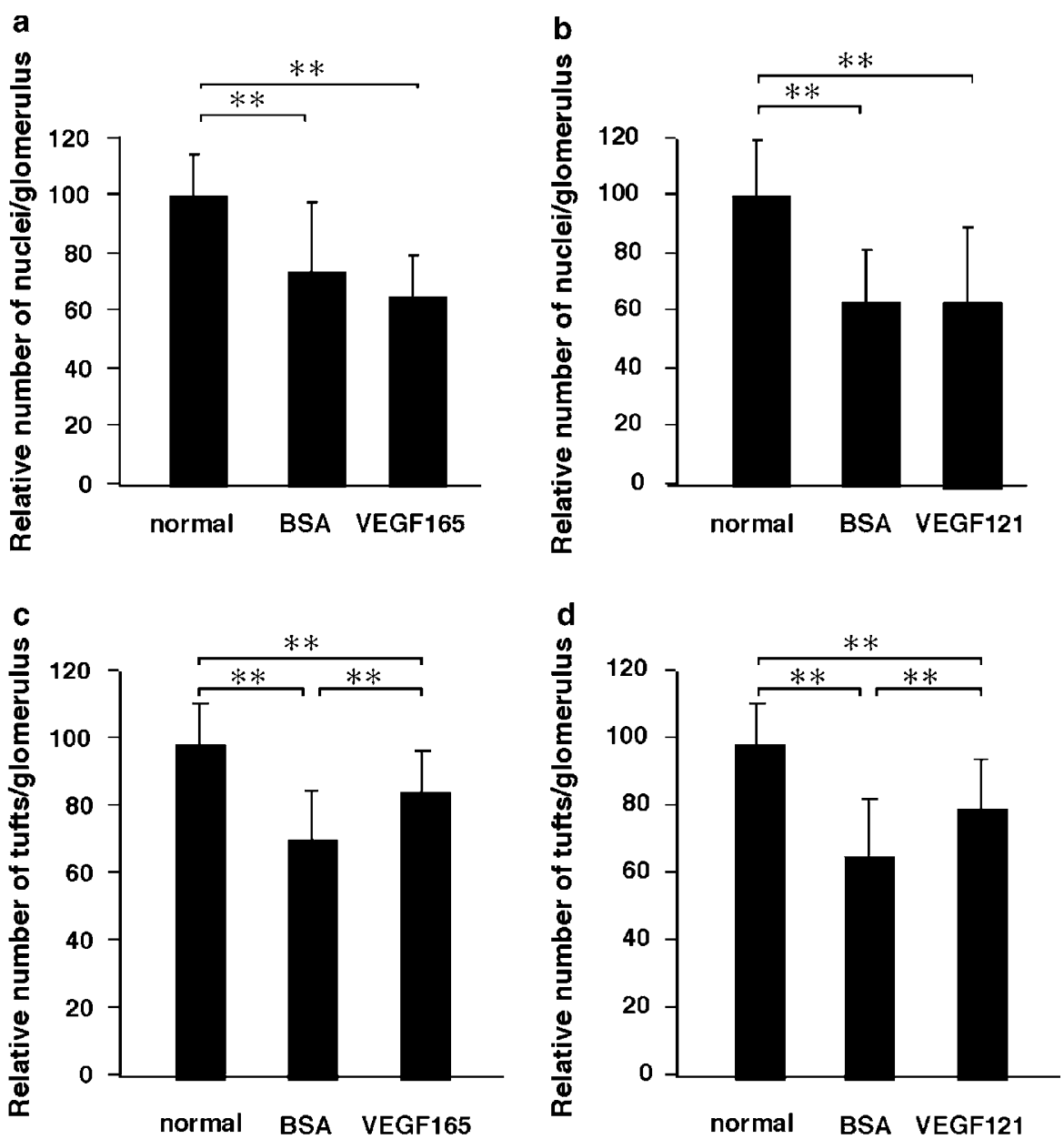

Figure 4 Protective effect of VEGF/VPF165 and VEGF/VPF121 on mesangial cell and endothelial cell injury during glomerulonephritis. Recombinant VEGF/VPF165 (a, c) or VEGF/VPF121 (b, d) was administered before injecting the anti-Thy-1.1 antibody, and number of nuclei $(\mathbf{a}, \mathbf{b})$ and number of tufts $(\mathbf{c}, \mathbf{d})$ per glomerular area on day 1 are shown. As a control, BSA was injected instead of VEGF/VPF. The number of nuclei and the number of tufts in glomeruli before the anti-Thy-1.1 antibody injection were used for normalization. Each experiment was repeated three times independently. ${ }^{*} P<0.01$.

\section{Assessment of renal function}

Serum creatinine concentration did not change throughout the course of glomerulonephritis. In control animals, the ratio of urinary protein to urinary creatinine was $0.69 \pm 0.16$ before the anti-Thy-1.1 antibody injection, and $3.77 \pm 1.83$ on day 4 . In VEGF/ VPF165-treated animals, the ratio was $1.11 \pm 0.30$ before the antibody injection and $1.58 \pm 0.65$ on day 4. Thus, urinary protein excretion on day 4 was suppressed to less than half by VEGF/VPF treatment.

\section{Expression of Flk-1 in the renal cortex}

On day 1, Flk-1 expression level in renal cortical tissue was suppressed by the anti-Thy-1.1 antibody injection, and further suppressed by VEGF/VPF165 (Figure 7a) or VEGF/VPF121 treatment (Figure 7b).

\section{Discussion}

In glomerulonephritis, induced in rats by injecting the monoclonal antibody to Thy-1.1, the degree of glomerular injury is dependent on the amount of injected antibody. By injecting a typical dose of antibody, severe mesangiolysis and ballooning of tufts were induced, changes rarely observed in human mesangial proliferative glomerulonephritis. To produce histological changes more compatible to those in human disease, we injected much less antibody and obtained glomerulonephritis lesions with relatively mild structural changes (Figure 1b-e) and without significant renal dysfunction. The changes were similar to glomerular changes seen in human disease.

VEGF/VPF165 or VEGF/VPF121 reduced the loss of capillary tufts but not of mesangial cells on day 1 after antibody injection (Figure 4), which indicates that either isoform prevents tuft dilation or rupture without preventing mesangiolysis (Figure 1f). This capillary protection may not be explained by angiogenic activity to maintain the microvasculature. ${ }^{15}$ Since glomerular endothelial apoptosis was not observed on day 1 under the present experimental condition, this protective effect cannot be explained 
by the antiapoptosis activity of $\mathrm{VEGF} / \mathrm{VPF}^{14,17,26}$ The VEGF/VPF activity to stimulate endothelial cells, reorganizing their cytoskeleton, ${ }^{27}$ may make

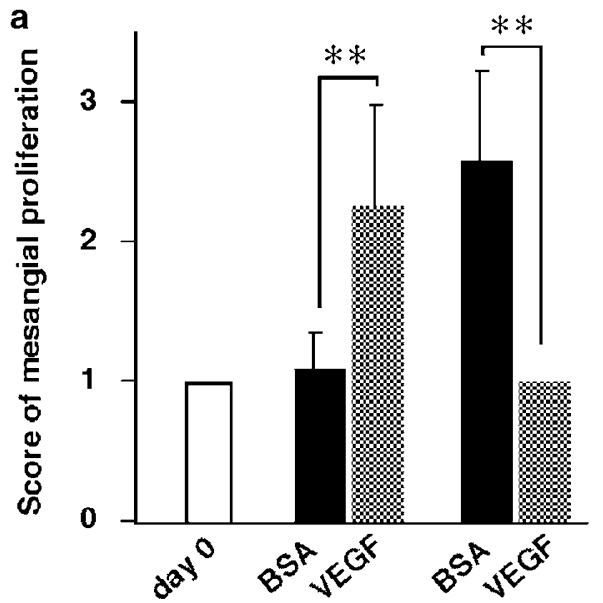

day 4

day 18

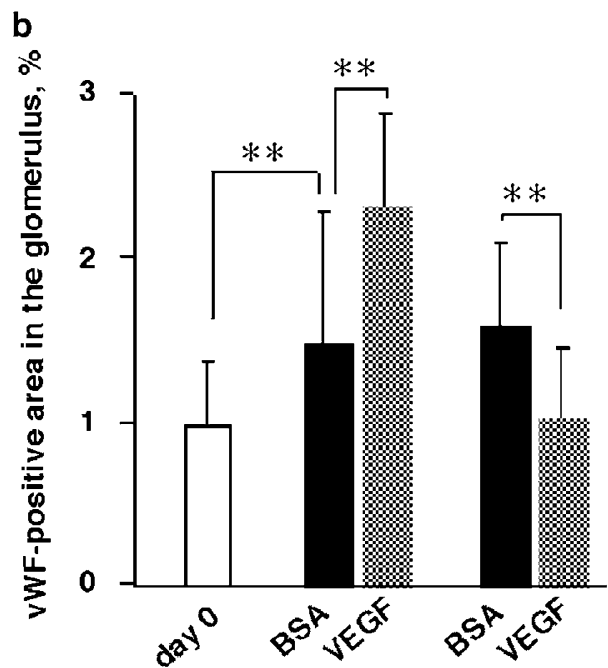

day 4 day 18

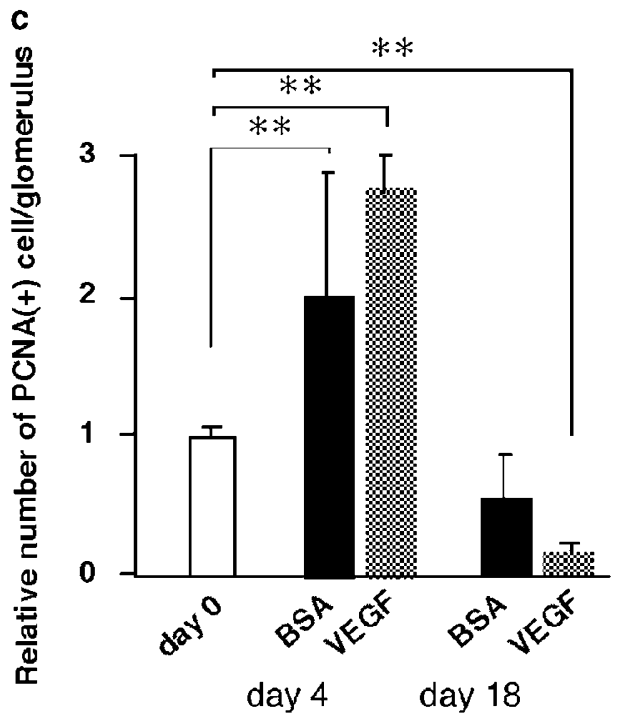

them more resistant to the stretching stress caused by loss of mesangial cells. The fact that the proliferation of endothelial cells was observed on day 4, when mesangial proliferation had not yet been present (Figure 5a,b), indicates that endothelial cell proliferation precedes mesangial proliferation. Since capillary structure can be a scaffold for the glomerular remodeling, this order of events may be essential for efficient repairing of injured glomeruli. The origin of mesangial cells committed to repair the lesion of mesangiolysis has not yet been identified. Mesangial cells might migrate to the lesion along the capillary from a vascular pole of the injured glomerulus or bone marrow-derived cells might migrate and differentiate in the lesion as reported recently. ${ }^{28}$ Whatever the origin is, preserved vessel structure is mandatory as an access for the cells to the lesion. The protection of endothelial cells by VEGF/VPF treatment caused an early onset of endothelial and mesangial cell proliferation, and resulted in early completion of repair as compared with the nontreated animals (Figure 1). This may explain the degree of proteinuria suppression caused by VEGF/VPF165 treatment. It is likely that the rate of glomerular cell repair inversely correlates to the degree of the initial endothelial injury. ${ }^{11}$

Besides proliferation,VEGF/VPF stimulates endothelial cells to secrete cytokines including plateletderived growth factor (PDGF) ${ }^{29}$ hepatocyte growth factor (HGF), ${ }^{23}$ nitric oxide (NO), ${ }^{30}$ and prostacyclin. ${ }^{31}$ PDGF stimulates mesangial cells to proliferate $^{32}$ and, in turn, activated mesangial cells secrete VEGF/VPF. HGF induces VEGF/VPF expression. ${ }^{33}$ NO and prostacyclin provide vascular protection. ${ }^{19}$ Thus, VEGF/VPF may protect glomerular endothelial cells in an indirect way, whereby positivefeedback mechanisms may operate.

VEGF/VPF isoforms administered intravenously stimulate the receptor Flk-1, located in the luminal side of the endothelial cells. ${ }^{34}$ The different binding affinities of isoforms to the matrix ${ }^{5}$ are not involved in this route of administration. The isoforms injected into the animals in the present study should result in plasma VEGF/VPF concentrations higher than $10 \mathrm{nM}$. This is at concentrations in which binding of the isoforms to recombinant Flk-1 is saturated. Therefore, the endothelial cell mitogenic activity is similar between VEGF/VPF165 and VEGF/VPF121 in our experimental condition; although the VEGF/VPF165 is more effective than

Figure 5 Effect of recombinant VEGF/VPF165 on the proliferation of the cells in the glomerulus during the course of glomerulonephritis. Recombinant VEGF/VPF165 or BSA was administered on the first 4 days of the experiment as described in the text. Mesangial proliferation score (a), vWF-positive area per glomerulus (b) and relative number of PCNA-positive cells per glomerulus (c) on day 0 (before the anti-Thy-1.1 antibody injection), day 4 and day 18 are shown. Each experiment was independently repeated three times. ${ }^{*} P<0.01$. 

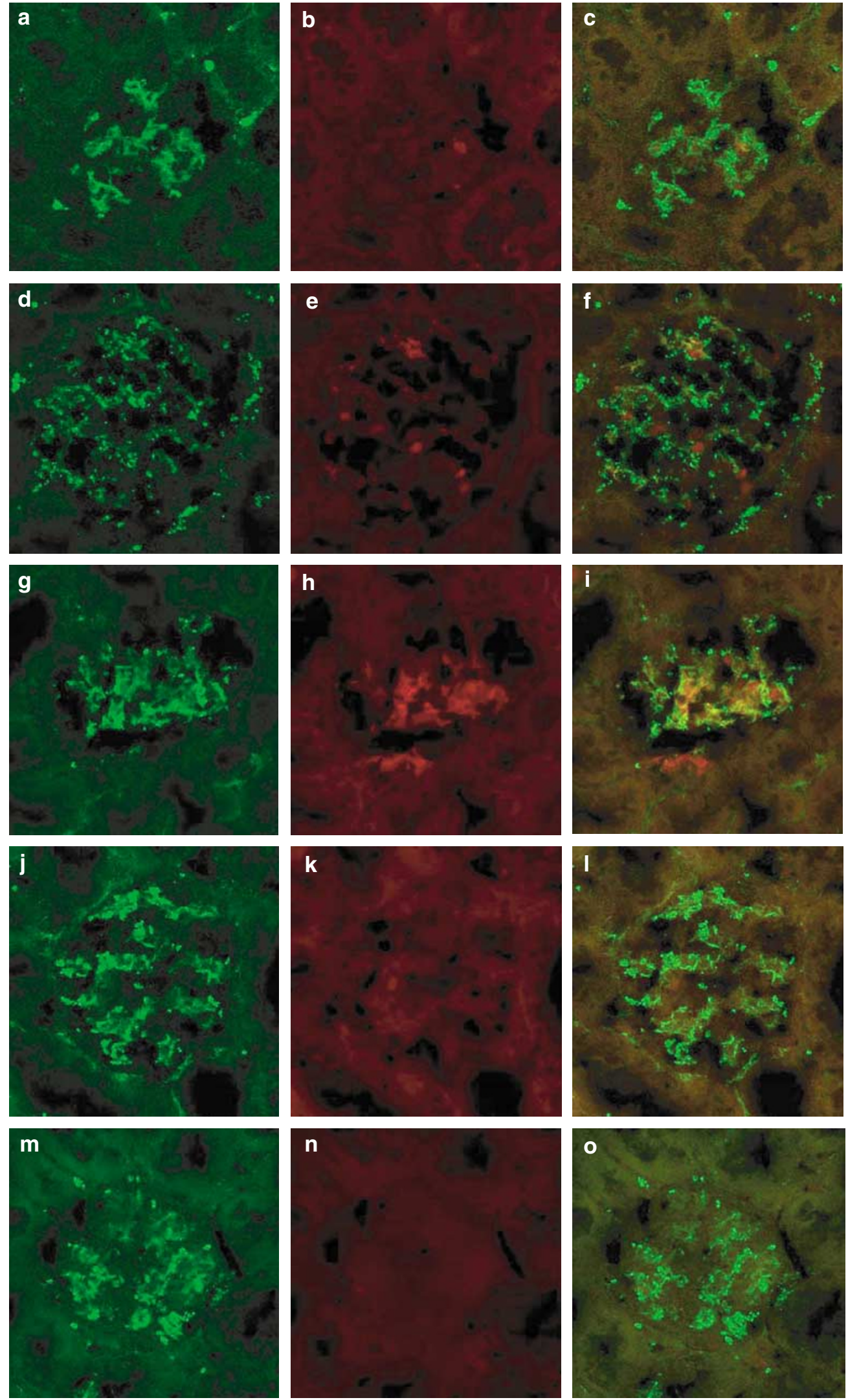

Figure 6 Changes of endothelial cells and proliferating cells during the course of glomerulonephritis. Immunofluorescent staining with vWF (green, left lane) and PCNA (red, middle lane), and merge of both the images (right lane) of a representative glomerulus before antiThy-1.1 antibody injection (a-c), and representative glomeruli in control (day $4(\mathbf{d}-\mathbf{f})$ and day 18 (j-l)) and VEGF/VPF-treated animals (day $4(\mathbf{g}-\mathbf{i})$ and day $18(\mathbf{m}-\mathbf{o})$ ) are shown (magnification $\times 140)$. 


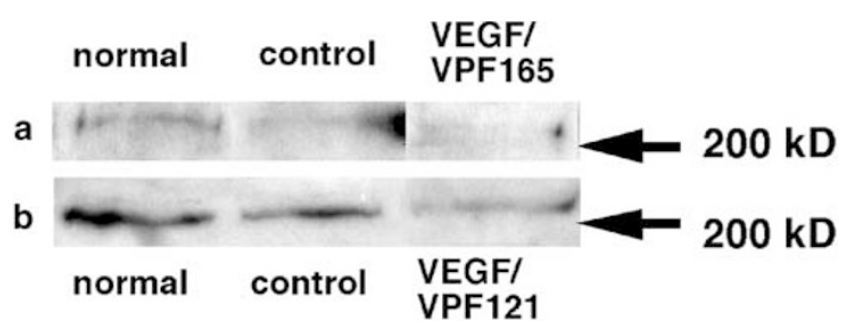

Figure 7 Suppression of Flk-1 expression in renal cortical tissues by VEGF/VPF165 (a) or VEGF/VPF121 (b) treatment. Densities of the Flk-1 bands decrease in the order of normal > control $>$ VEGF/ VPF. Normal, control and VEGF/VPF represent the supernatants of homogenates from the animal before the antibody injection, from the control animal on day 1, and from the VEGF/VPF-treated animal on day 1 , respectively.

the smaller isoform at lower concentrations. As intrinsic VEGF/VPF isoform concentrations in the blood are less than $30 \mathrm{pg} / \mathrm{ml}$ and administered VEGF/VPF concentration is initially higher than $1 \mu \mathrm{g} / \mathrm{ml}$, the Flk-1 at the luminal side of endothelial cells is presumably occupied by the administered VEGF/VPF isoforms. The Flk-1 expression level in the renal cortex was equally suppressed by VEGF/ VPF165 and VEGF/VPF121. Therefore, it may be relevant that VEGF/VPF165 and VEGF/VPF121 intravenous administrations equally protected glomerular endothelial cells (Figure 4). To compare the protective activities of the two isoforms more precisely, further studies using lower concentrations of the isoforms would be necessary.

\section{Conclusion}

This is the first study to show that VEGF/VPF121 and VEGF/VPF165 equally protect glomerular endothelial cells against injury by an apoptosisindependent mechanism. This protection might be applicable as a therapeutic strategy in renal diseases.

\section{Acknowledgements}

We thank Dr J Evan Sadler and Dr Ivette Revollo for useful discussions. We also thank Miss Noriko Teramoto and Miss Yasuyo Hayashida for their technical assistance. This work was supported by Grant-in-Aid for Scientific Research from Japanese Ministry of Education and Science (13671120 to YK).

\section{References}

1 Ferrara N. Vascular endothelial growth factor: molecular and biological aspects. Curr Top Microbiol Immunol 1999;237:1-30.

$2 \mathrm{Ng}$ YS, Rohan R, Sunday ME, et al. Differential expression of VEGF isoforms in mouse during development and in the adult. Dev Dyn 2001;220: 112-121.

3 Gitay-Goren H, Cohen T, Tessler S, et al. Selective binding of VEGF121 to one of the three vascular endothelial growth factor receptors of vascular endothelial cells. J Biol Chem 1996;271:5519-5523.

4 Keyt BA, Berleau LT, Nguyen HV, et al. The carboxylterminal domain (111-165) of vascular endothelial growth factor is critical for its mitogenic potency. J Biol Chem 1996;271:7788-7795.

5 Houck KA, Leung DW, Rowland AM, et al. Dual regulation of vascular endothelial growth factor bioavailability by genetic and proteolytic mechanisms. J Biol Chem 1992;267:26031-26037.

6 Soker S, Takashima S, Miao HQ, et al. Neuropilin-1 is expressed by endothelial and tumor cells as an isoform-specific receptor for vascular endothelial growth factor. Cell 1998;92:735-745.

7 Guo P, Xu L, Pan S, et al. Vascular endothelial growth factor isoforms display distinct activities in promoting tumor angiogenesis at different anatomic sites. Cancer Res 2001;61:8569-8577.

8 Carmeliet P, Ng YS, Nuyens D, et al. Impaired myocardial angiogenesis and ischemic cardiomyopathy in mice lacking the vascular endothelial growth factor isoforms VEGF164 and VEGF188. Nat Med 1999;5:495-502.

9 Mattot V, Moons L, Lupu F, et al. Loss of the VEGF164 and VEGF188 isoforms impairs postnatal glomerular angiogenesis and renal arteriogenesis in mice. J Am Soc Nephrol 2002;13:1548-1560.

10 Pabst R, Sterzel RB. Cell renewal of glomerular cell types in normal rats. An autoradiographic analysis. Kidney Int 1983;24:626-631.

11 Shimizu A, Kitamura H, Masuda Y, et al. Rare glomerular capillary regeneration and subsequent capillary regression with endothelial cell apoptosis in progressive glomerulonephritis. Am J Pathol 1997; 151:1231-1239.

12 Alon T, Hemo I, Itin A, et al. Vascular endothelial growth factor acts as a survival factor for newly formed retinal vessels and has implications for retinopathy of prematurity. Nat Med 1995;1:1024-1028.

13 Benjamin E, Keshet E. Conditional switching of vascular endothelial growth factor (VEGF) expression in tumors: induction of endothelial cell shedding and regression of hemangioblastoma-like vessels by VEGF withdrawal. Proc Natl Acad Sci USA 1997;94: 8761-8766.

14 Gerber HP, Dixit V, Ferrara N. Vascular endothelial growth factor induces expression of the antiapoptotic proteins Bcl-2 and A1 in vascular endothelial cells. J Biol Chem 1998;273:13313-13316.

15 Kang DH, Hughes J, Mazzali M, et al. Impaired angiogenesis in the remnant kidney model: II. Vascular endothelial growth factor administration reduces renal fibrosis and stabilizes renal function. J Am Soc Nephrol 2001;12:1448-1457.

16 Kelly DJ, Hepper C, Wu LL, et al. Vascular endothelial growth factor expression and glomerular endothelial cell loss in the remnant kidney model. Nephrol Dial Transplant 2003;18:1286-1292.

17 Ostendorf T, Kunter U, Eitner F, et al. VEGF(165) mediates glomerular endothelial repair. J Clin Invest 1999;104:913-923.

18 Masuda Y, Shimizu A, Mori T, et al. Vascular endothelial growth factor enhances glomerular capillary 
repair and accelerates resolution of experimentally induced glomerulonephritis. Am J Pathol 2001;159: 599-608.

19 Zachary I, Mathur A, Yla-Herttuala S, et al. Vascular protection : a novel nonangiogenic cardiovascular role for vascular endothelial growth factor. Arterioscler Thromb Vasc Biol 2000;20:1512-1520.

20 Castilla MA, Caramelo C, Gazapo RM, et al. Role of vascular endothelial growth factor (VEGF) in endothelial cell protection against cytotoxic agents. Life Sci 2000;67:1003-1013.

21 Kuzuya M, Ramos MA, Kanda S, et al. VEGF protects against oxidized LDL toxicity to endothelial cells by an intracellular glutathione-dependent mechanism through the KDR receptor. Arterioscler Thromb Vasc Biol 2001;21:765-770.

22 Jin KL, Mao XO, Greenberg DA. Vascular endothelial growth factor: direct neuroprotective effect in in vitro ischemia. Proc Natl Acad Sci USA 2000;97: 10242-10247.

23 LeCouter J, Moritz DR, Li B, et al. Angiogenesisindependent endothelial protection of liver: role of VEGFR-1. Science 2003;299:890-893.

24 Ishizaki M, Masuda Y, Fukuda Y, et al. Experimental mesangioproliferative glomerulonephritis in rats induced by intravenous administration of antithymocyte serum. Acta Pathol Jpn 1986;36: 1191-1203.

25 Iyonaga $\mathrm{K}$, Takeya $\mathrm{M}$, Yamamoto $\mathrm{T}$, et al. A novel monoclonal antibody, RM-4, specifically recognizes rat macrophages and dendritic cells in formalinfixed, paraffin- embedded tissues. Histochem J 1997;29:105-116

26 Watanabe Y, Dvorak HF. Vascular permeability factor/ vascular endothelial growth factor inhibits anchoragedisruption-induced apoptosis in microvessel endothelial cells by inducing scaffold formation. Exp Cell Res 1997;233:340-349.
27 Enomoto T, Okamoto T, Sato JD, et al. Vascular endothelial growth factor induces the disorganization of actin stress fibers accompanied by protein tyrosine phosphorylation and morphological change in Balb/ C3T3 cells. Biochem Biophys Res Commun 1994;202: 1716-1723.

28 Imasawa $\mathrm{T}$, Utsunomiya $\mathrm{Y}$, Kawamura $\mathrm{T}$, et al. The potential of bone marrow-derived cells to differentiate to glomerular mesangial cells. J Am Soc Nephrol 2001; 12:1401-1409.

29 Collins T, Ginsburg D, Boss JM, et al. Cultured human endothelial cells express platelet- derived growth factor B chain: cDNA cloning and structural analysis. Nature 1985;316:748-750.

$30 \mathrm{Ku} \mathrm{DD}$, Zaleski JK, Liu S, et al. Vascular endothelial growth factor induces EDRF- dependent relaxation in coronary arteries. Am J Physiol 1993;265:H586-H592.

31 Wheeler-Jones C, Abu-Ghazaleh R, Cospedal R, et al. Vascular endothelial growth factor stimulates prostacyclin production and activation of cytosolic phospholipase A2 in endothelial cells via p42/p44 mitogen-activated protein kinase. FEBS Lett 1997;420: 28-32.

32 Shultz PJ, DiCorleto PE, Silver BJ, et al. Mesangial cells express PDGF mRNAs and proliferate in response to PDGF. Am J Physiol 1988;255:F674-F684.

33 Van Belle E, Witzenbichler B, Chen D, et al. Potentiated angiogenic effect of scatter factor/hepatocyte growth factor via induction of vascular endothelial growth factor: the case for paracrine amplification of angiogenesis. Circulation 1998;97:381-390.

34 Feng D, Nagy JA, Brekken RA, et al. Ultrastructural localization of the vascular permeability factor/vascular endothelial growth factor (VPF/VEGF) receptor-2 (FLK-1, KDR) in normal mouse kidney and in the hyperpermeable vessels induced by VPF/VEGFexpressing tumors and adenoviral vectors. J Histochem Cytochem 2000;48:545-556. 